\title{
Language Acquisition
}

\section{Introduction}

Studies of Papuan languages played an early and important role in the emergence and development of the language socialization paradigm within linguistic anthropology: ${ }^{1}$ especially Kaluli (e.g., Schieffelin 1990), but also Huli (e.g., Goldman 1987) and Tayap (e.g., Kulick 1992). Around the same time, Dan Slobin and colleagues promoted The Crosslinguistic Study of Language Acquisition (Slobin 1985-1997), adopting a psycholinguistically-oriented perspective on acquisition, but also integrating anthropological perspectives. Again, the study of a Papuan language, Kaluli, contributed to this crosslinguistic project (Schieffelin 1985). Since then, however, there have been few studies of language acquisition and socialization in under-documented languages, despite an awareness of the need to diversify acquisition data (as seen in the ACQDIV project, Jansco et al. 2020; see also the urgent call by Pye 2021). As Kelly and Nordlinger (2014: 180) put it: “despite the promise of this earlier typologically diverse work, when we fast-forward 30 years we do not see a boom in the study of FLA [First Language Acquisition] in typologically diverse languages and culturally different communities [...].” Their remark also applies to Papuan languages, and it is only over the last decade that a renewed interest in Papuan language acquisition emerged, with projects targeting the acquisition of Duna, Kaluli, Ku Waru, Nungon, Qaqet (see also Hellwig et al., this volume) and Yélî Dnye (see also Levinson, this volume).

Table 1 lists the Papuan languages for which acquisition or socialization studies exist. In this chapter, we focus on acquisition research, and we refer the reader to San Roque \& Schieffelin (this volume) for discussion of socialization research.

Table 1. Acquisition and socialization research in Papuan languages

\begin{tabular}{llll}
\hline Language & Glottolog & Classification & Researchers \\
\hline Duna & duna1248 & Duna-Bogaya & San Roque \\
Huli & huli1244 & Engan & Goldman \\
Kaluli (Bosavi) & kalu1248 & Bosavi & Schieffelin, San Roque \\
Ku Waru (Mbo-Ung) & boun1245 & Chimbu-Wahgi & Rumsey, Merlan, Reed \\
Nungon & yaum1237 & Finisterre-Huon & Sarvasy \\
Qaqet & qaqe1238 & Baining & Hellwig, Dawuda, Frye, Reetz \\
Tayap & taia1239 & isolate & Kulick \\
Yélî Dnye & yele1255 & isolate & Casillas, Brown, Levinson, \\
& & & Cristia, Peute \\
\hline
\end{tabular}

\footnotetext{
${ }^{1}$ Other languages of the Pacific region also played important roles, including the Polynesian language Samoan (Ochs 1988) as well as the Oceanic language Kawara’ae (Watson-Gegeo \& Gegeo 1986).
} 
Acquisition research in Papuan languages has a strong focus on spontaneous language use within naturalistic settings. Experimental research, by contrast, has so far played a much more limited role (limited to on-going work in Yélî Dnye, to our knowledge), but may assume a more central role in the future, as more naturalistic baseline data becomes available. A strong foundation of naturalistic data will yield an idea of the developmental stages and approximate ages at which we can expect specific structures to appear and become productive. For Papuan languages, this knowledge needs to be built from scratch, and the only holistic overview of (early) developmental stages has been Schieffelin's (1985) sketch of the acquisition of Kaluli.

Table 2 summarizes the existing data sources on Papuan languages. Note that most corpora are still under construction, with only parts of the data transcribed and annotated. There exist two types of corpora: longitudinal corpora where a small number of children are recorded at regular intervals over a long period, and cross-sectional corpora where a large number of children of different ages are recorded at a few points in time. Both approaches address the issue of individual variation, as there is considerable variation in children's development, making it difficult to generalize across children (Kidd \& Donnelly 2020). In a longitudinal setup, the response to individual variation is to investigate development for each child individually. In a cross-sectional setup, the response is to sample a large number of children. While language development begins before age 2 and continues into adolescence, the period between 2 and 5 is when most major grammatical structures are acquired. All of the projects listed here strive to at least cover some of this period. Depending on the research question and available resources, younger and/or older children may be included.

Table 2. Child language corpora of Papuan languages ${ }^{2}$

\begin{tabular}{|c|c|c|c|}
\hline Language & Type of data & $\begin{array}{l}\text { Children (approx. } \\
\text { age range) }\end{array}$ & Archived \\
\hline Duna & $\begin{array}{l}\text { cross-sectional: various interactions } \\
\text { with children, video (2010) }\end{array}$ & $\begin{array}{l}5 \text { children } \\
(1 ; 3-3 ; 2)^{3}\end{array}$ & $\begin{array}{l}\text { San Roque } \\
2003\end{array}$ \\
\hline Kaluli & $\begin{array}{l}\text { longitudinal: 3 hrs. per 4-6 weeks, } \\
\text { audio (1975-1976) }\end{array}$ & $\begin{array}{l}3 \text { children } \\
(2 ; 0-2 ; 11)\end{array}$ & $\mathrm{n} / \mathrm{a}$ \\
\hline \multirow[t]{2}{*}{ Ku Waru } & $\begin{array}{l}\text { longitudinal: } \sim 40 \text { min. at irregular } \\
\text { intervals, audio (2004-2006) }\end{array}$ & $\begin{array}{l}2 \text { children } \\
(1 ; 8-3 ; 1)\end{array}$ & \multirow[t]{2}{*}{ Rumsey 1983} \\
\hline & $\begin{array}{l}\text { longitudinal: } \sim 1 \text { hr. per month, video } \\
\text { (2013-2016) }\end{array}$ & $\begin{array}{l}5 \text { children } \\
(2 ; 2-4 ; 10)\end{array}$ & \\
\hline \multirow[t]{2}{*}{ Nungon } & $\begin{array}{l}\text { longitudinal: } ~ 1 \text { hr. per month, video } \\
\text { (2015-2017) }\end{array}$ & $\begin{array}{l}5 \text { children } \\
(1 ; 1-5 ; 8)\end{array}$ & \multirow[t]{2}{*}{ Sarvasy 2017} \\
\hline & $\begin{array}{l}\text { longitudinal: } \sim 4 \text { hrs. per month } \\
\text { (within a single week), video ( } 5 \\
\text { months in 2019; further recording } \\
\text { planned for 2021) }\end{array}$ & $\begin{array}{l}5 \text { children } \\
(2 ; 3-2 ; 11)\end{array}$ & \\
\hline Qaqet & $\begin{array}{l}\text { longitudinal: 1 hr. per week, video } \\
\text { (2014-2018) }\end{array}$ & $\begin{array}{l}6 \text { children } \\
(1 ; 11-4 ; 7)\end{array}$ & $\begin{array}{l}\text { Hellwig et al. } \\
2014\end{array}$ \\
\hline
\end{tabular}

\footnotetext{
${ }^{2}$ In addition, there exist corpora collected within language socialization research, consisting of interview data and various interactions with adults and children. We are aware of the Tayap corpus (archived; Kulick 1980) and the Huli corpus (mentioned in Goldman 1987).

${ }^{3}$ Ages of children are given in the format YEAR;MONTH, e.g., 3;2 means an age of 3 years and 2 months.
} 


\begin{tabular}{|c|c|c|c|}
\hline \multirow[t]{2}{*}{ Yélî Dnye } & $\begin{array}{l}\text { cross-sectional: daylong recordings, } \\
\sim 8-9 \text { hrs. per recording, one } \\
\text { recording per child, image-linked } \\
\text { audio (2016) }\end{array}$ & $\begin{array}{l}57 \text { children } \\
(0 ; 0-4 ; 0)\end{array}$ & $\begin{array}{l}\text { Casillas et al. } \\
2017\end{array}$ \\
\hline & $\begin{array}{l}\text { cross-sectional; daylong recordings, } \\
\sim 16-24 \text { hrs. per recording, up to three } \\
\text { recordings per child, audio (2019) }\end{array}$ & $\begin{array}{l}45 \text { children } \\
(0 ; 3-6 ; 4)\end{array}$ & $\begin{array}{l}\text { Cristia \& } \\
\text { Casillas } 2020\end{array}$ \\
\hline
\end{tabular}

In this chapter, we review research on the acquisition of Papuan languages, with a special focus on the kinds of contributions that studies of Papuan languages can make to language acquisition research in general. Section 2 highlights salient properties of Papuan cultures and languages that are particularly interesting from a developmental perspective and gives a brief overview of existing studies on the acquisition of Papuan languages. Sections 3 to 6 present case studies, outlining the state of research on the acquisition of phonology (section 3), clause chaining (section 4) and complex predicates (section 5). Section 6 concludes this chapter with an outlook on future developments.

\section{Topics in the acquisition of Papuan languages}

Theories of language development are grounded in empirical data from a small number of languages, mostly biased towards the major European languages (Kelly \& Nordlinger 2014; Pye 2021). Extending language acquisition research to languages of other regions is crucial, and time is running out to do this for many languages of the world. For Papua New Guinea, Kik et al. (2021) found that only 58\% of secondary school students are fluent in at least one indigenous language (Papuan or Austronesian, excluding Tok Pisin), and this is predicted to decrease to about $26 \%$ in the next generation of secondary students.

Sarvasy (2021b) distinguishes two complementary approaches for extending acquisition research to more varied languages and cultures (see also Sarvasy \& Tuninetti, this volume, for psycholinguistic research with adults). In one approach, articles seek to replicate research that was originally done on English or other European languages in order to, e.g., pry apart two competing theories (Cutler 1985), or to validate assumptions based on English. In another approach, studies target language-specific features, regardless of whether they are part of the 'theoretical canon' found in acquisition textbooks (Pye 2021), with the goal of thoroughly describing their acquisition, only afterward relating the findings to existing literature. Most studies of the acquisition of Papuan languages fall into the second category. The reasons here are manifold: the training in field linguistics or linguistic anthropology (rather than laboratory psycholinguistics) of most of the active researchers; the presence of salient language- and culture-specific features that play no or only a minor role in some of the major languages; or the focus on creating naturalistic corpora and establishing larger developmental trajectories before homing in on more specific issues. ${ }^{4}$

Regardless of the chosen approach, there are characteristics of Papuan cultures and languages that make them of special interest to the study of language acquisition.

Starting with the cultural contexts, a considerable body of research shows that input from adults directed at children (not overheard by children) facilitates language learning (Shneidman \& Woodward 2016). In Anglophone North America and Western Europe, child-

\footnotetext{
${ }^{4}$ That said, Sarvasy (2019) is a contribution premised on engagement with the theoretical canon on 'root' or 'optional' infinitives in child language.
} 
directed interaction is said to predominate. In Melanesia (and many other parts of the world), by contrast, qualitative descriptions of interactional contexts indicate that multi-party interactions are widespread (Schieffelin \& San Roque, this volume). In addition, there exists one large-scale quantitative study on the input to Yélî Dnye children under age 3;0 (Casillas et al. 2021). This study indeed shows child-directed interaction to be infrequent (an average of 3.13 minutes per hour), but also found some bursts during the day (increasing to 14.45 minutes per hour during interactional peaks) (see also Casillas et al. 2020 and Cristia et al. 2019 for comparable findings from other subsistence-based communities outside of Melanesia). Given the assumptions in much of the literature that direct adult input is best for learning, it could be presumed that the Yélî Dnye children might evince delays in language development. On the contrary, Casillas et al. (2021) found that the children achieve all linguistic milestones, despite limited child-directed input. Furthermore, recent research from other parts of the world suggests that children can adjust to their learning environments. For example, children may be better at learning from non-directed interaction if they experience little directed interaction in their everyday life (see especially Shneidman \& Woodward 2016 for a review of available research). Another important type of interaction across Melanesia is peer interaction. Peers are usually considered more challenging conversational partners than adults, but little is known about the impact of peer interaction on the acquisition of language (Barton \& Tomasello 1994; Lieven 1994).

Given the predominance of multiparty and peer interaction in the Papuan region, the study of Papuan languages can thus contribute important insights to debates on learning strategies. This includes quantitative studies along the lines of the Yélî Dnye input study, but also qualitative studies on the specific properties of the language used with and around children. San Roque \& Schieffelin (this volume) give a concise overview of this topic: the availability of a child-directed language register and its properties in several Papuan languages, but also its absence in others; as well as the availability of linguistic routines, such as prompting, that facilitates the participation of young children in multiparty interactions.

Turning now from the contexts of learning to the typological structures of the languages being learned, Papuan languages exhibit phenomena that are not yet included in textbooks on language acquisition. These include clause chaining and switch-reference (see section 4); complex lexical expressions that exhibit varying degrees of compositionality and transparency (see section 5); and phenomena that show formal or semantic peculiarities that can offer new insights into the acquisition of better-researched topics, such as phonological systems (see section 3), but also optional ergativity and egophoricity.

Rumsey et al. (2013) address the acquisition of ergative case marking in Kaluli, Ku Waru and Duna. Ergativity is often assumed to present special challenges to the child, especially because ergative structures tend to co-exist with non-ergative structures in the form of, e.g., split ergativity, optional ergativity, and/or ergative case marking interacting with nominative/accusative verb indexing (see NN, this volume, for an overview of Papuan case systems). But while there exists extensive research on the acquisition of case in nominative/accusative systems, there is little corresponding research on ergativity (see especially Bavin \& Stoll 2013), and there remain many open questions as to how children acquire the complexities of the system. Rumsey et al.'s comparative analysis shows that children are sensitive to the language-specific peculiarities early on. They tend to first use ergative marking in frequent and (near-)obligatory contexts, gradually extending it to other contexts. In all cases, their error patterns reflect the peculiarities of the different systems. For Kaluli children, the difficulty lies in the interaction of case marking with constituent order and animacy constraints (see also Schieffelin 1985; Rispoli 1991); for Ku Waru children, it is the distribution of ergative marking in intransitive contexts; and for Duna children, it is the 
distribution in verbless clauses. Despite language-specific differences in usage contexts and morphological complexity, children seem to acquire the systems at approximately the same pace, with adult-like structures being mastered around the age of 3;0.

Another topic concerns the acquisition of egophoricity, which has been investigated for Kaluli (San Roque \& Schieffelin 2018) and Ku Waru (Rumsey 2020; see also Rumsey 2003; 2013; 2019 on the acquisition of a range of phenomena belonging to the broader domain of intersubjectivity). As San Roque \& Schieffelin (2018: 441) remark, studying the acquisition of such systems may eventually give insights "into how the development of sophisticated theory of mind abilities ties in with linguistic structure [...].” The two Papuan languages do not have typical egophoric or conjunct/disjunct systems, but they do have structures that convey the speaker's (in declarative sentence types) or addressee's (in interrogative sentence types) epistemic authority over an event (see also San Roque, this volume). This information is expressed within the TAM systems of the two languages, which are sensitive not only to person/number categories, but also - to some extent - to knowledge status. I.e., children acquiring TAM inflections also need to learn about egophoricity. For the moment, available research focuses on the input and investigates how these markers are used to manage epistemic authority in interaction with children. Preliminary insights into child language are emerging, though. For Kaluli, for example, error patterns reveal problems with 1st person contexts in the present tense (but not in the future tense) as well as with the marking of second-person questions. Future investigations will be able to (dis)confirm these patterns and to suggest possible explanations.

Other available research exists on properties of child-directed language (e.g., Frye 2019; Hellwig \& Jung 2020; Rumsey et al., in prep.; Sarvasy, under review; Sarvasy et al. 2019), the acquisition of non-finite verb forms in Nungon (Sarvasy 2019), and on perception verbs in Kaluli (San Roque \& Schieffelin 2019).

\section{Phonology}

Papuan languages are not generally known for their complex phoneme inventories (Foley 1986: 55; but see Hajek, this volume). That said, there is minimally one widespread phenomenon that may prove to be of interest to language acquisition research: the acquisition of a voicing contrast in plosives, and especially the role of prenasalization. Ongoing phonetic work on adult Qaqet shows an exceptionally long voicing period for voiced plosives, which can (but need not) be maintained by means of prenasalization (Tabain \& Hellwig, to appear; Tabain et al., in prep.). Such long voicing periods are typologically unusual and not well understood, neither in terms of their phonetics nor in terms of their acquisition. Given that prenasalization is widespread across Papuan (and Oceanic) languages, it is possible that long voicing periods may be more widespread, too. To date, there is no systematic study of the acquisition of this phenomenon, but there are indications that Qaqet children acquire it fairly late, with non-target-like realizations attested well into 3-4 years of age.

In addition, the phoneme inventories of individual Papuan languages differ considerably, including some with typologically rare features that present children with interesting puzzles to solve. Ku Waru, for example, distinguishes four laterals: a retroflex lateral flap /J/, an alveolar lateral continuant $/ \mathrm{l} /$, a palatal lateral continuant $/ K /$, and a pre-stopped velar lateral $/{ }^{1} \mathrm{~L} /$. Rumsey (2017: 97-102) discusses aspects of their acquisition, focusing on the late acquisition of the complex phoneme $/{ }^{/ 9} \mathrm{~L} /$ (with target-like realizations around 5-6 years of age), and the common realization of this phoneme as $[1]$ in child-directed language. 
Among the many different phoneme inventories of Papuan languages, it is probably the one of Yélî Dnye that presents children with most challenges. With 56 contrastive consonants and 34 contrastive vowels, the inventory itself is rather large (Levinson, to appear; see Figure 1). Despite its size, it is acoustically packed, the combinatorial outcome of a few key parameters. For consonants, these parameters include four primary places of articulation (labial, prealveolar, post-alveolar, and velar), oral vs. nasal airflow, single- or double-articulation, and pre-nasalization or nasal release with labialized, palatalized, or labial-palatal release coloring; there is no contrastive voicing and only 7 of these phones are non-nasal continuants. For vowels, these parameters include 10 basic qualities, 7 of which have contrastive oral vs. nasal airflow, and all 17 of which contrast short vs. long duration. Already a formidable challenge to the language learner because of its densely packed acoustic space, this phonological inventory has a few features that are rare across the world's languages. Consider, for example, the complex contrastive use of nasalization (e.g., oral vs. nasal contrast in consonants and vowels and pre-nasalization or nasal release), combined with the possibility of labial and palatal release.

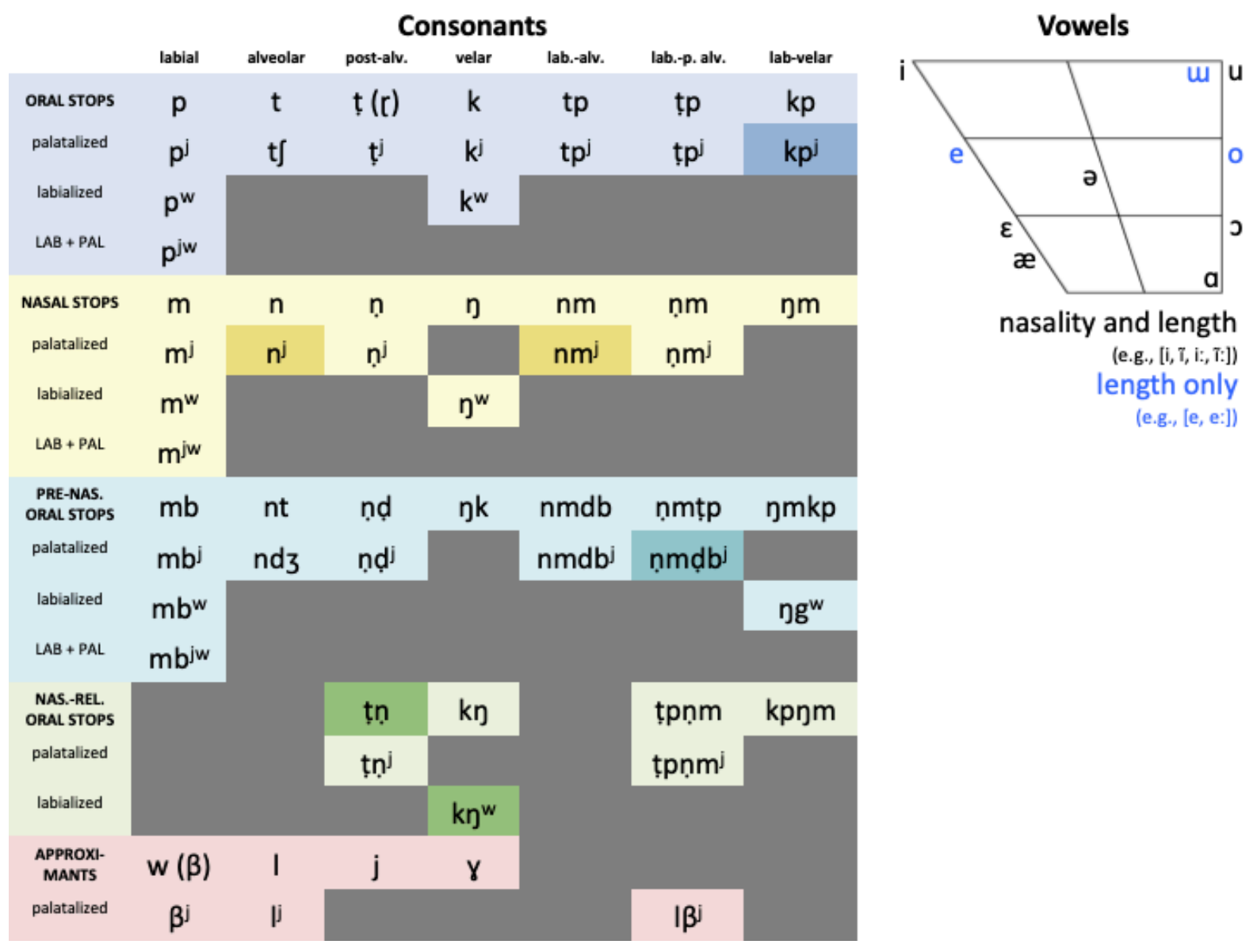

Figure 1. An overview of the Yélî Dnye phonological inventory. Consonants shown between parentheses represent intervocalic allophones. Consonants shown in a darker shade of their segmental type (e.g., $\mathrm{kp}^{\mathrm{j}}$ ) are contrastively attested in minimal pairs but are rare in usage. 
It is an open question how such acoustic density and typological markedness would impact children's phonological development (but see Sundara et al. 2018 on subtle phonetic contrasts). Recent and on-going work ${ }^{5}$ on the acquisition of Yélî Dnye hints at a protracted developmental trajectory, both in production and comprehension processes related to the native phonology. Specifically, both early and late production and perception patterns suggest that children's mastery of this sound system takes place over a long period that is initially biased toward segments and segmental contrasts that are frequently found across the world's languages, with only later mastery of the rarer segments and contrasts contained within the native phonology.

This includes evidence from the pre-lexical and early lexical phase: Peute \& Casillas (in revision) found no evidence for early production of the complex consonants mentioned above in the spontaneous pre-lexical and early lexical vocalizations from 12 children between ages $0 ; 8$ and 1;5; and Casillas (in prep.) found no evidence for perceptual discrimination between $/ t /$ and $/ t$ / in 26 children between ages 0;6 and 1;2. In other work, Casillas and Levinson (in prep.) have begun to investigate how the effects of typological markedness influence word recognition in older children. They asked Yélî Dnye-acquiring children between ages 3;0 and $12 ; 0$ to listen to a word and then choose its matching image from an image pair featuring that

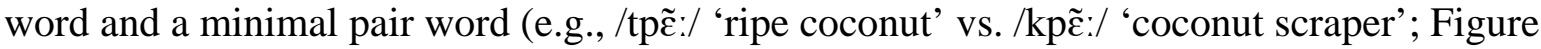
2). Minimal pairs included the basic oral stop consonants, with some pairs featuring typologically rare (e.g., simplex /t/ vs. /ț/ and complex /tp/ vs. /țp/) and typologically frequent place of articulation contrasts (e.g., simplex /t/ vs. /k/ and complex /tp/ vs. /kp/). Children's response patterns on the minimal pairs improved in accuracy with age, from chance at 3;0 to ceiling at 12;0. However, the rate of improvement was faster for frequent vs. rare place of articulation contrasts, supporting the idea that, while all native to the language, the rare contrasts show a marked developmental trajectory. Adults tested using the same experimental paradigm do not show this effect, suggesting that speakers do resolve these perceptual differences some time during or after adolescence.

\footnotetext{
${ }^{5}$ In all of these studies, participants were primarily acquiring and hearing the dialect of Yélî Dnye spoken around the Jinjo area of Rossel Island (i.e., the northeastern region).
} 


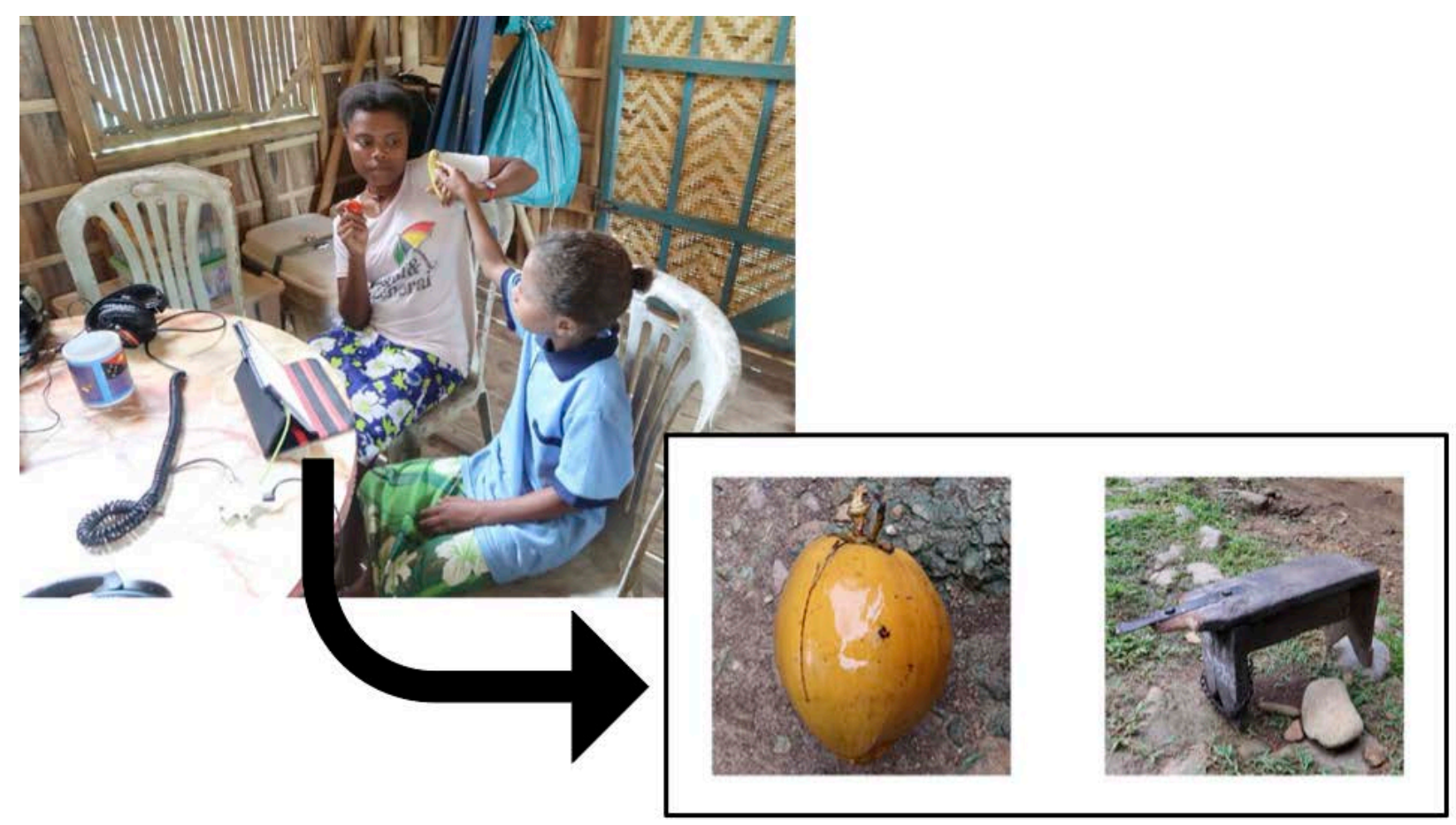

Figure 2. Example image of a real-world object training trial (left) and a computer-tablet test trial (right) from Casillas \& Levinson (in prep.).

\section{Clause chaining}

Papuan languages are known for clause chaining (see Sarvasy, this volume), in which sequences of events or actions are described with one or more clauses with non-finite, "medial verb," predicates, followed by a single “final” clause with a finite, “final,” predicate. Most Papuan languages with clause chaining include switch-reference marking on medial verbs (see Daniels, this volume). Switch-reference markers announce, in advance, whether the subject of the upcoming clause will be the same or different from that of the current clause.

Although some relatively well-studied languages, including Japanese, Korean, and Turkish, feature clause chains, child language researchers did not target clause chains for investigation in their own right until a recent volume covering the acquisition of clause chaining across several languages, including the Papuan languages Ku Waru and Nungon (Sarvasy \& Choi 2020a). Major points of interest for child acquisition of clause chaining across all languages are: child clause chain length, semantics, and, for those languages with it, switch-reference marking.

Of the Papuan languages with major, searchable corpora of child-caregiver interactions, only $\mathrm{Ku}$ Waru and Nungon feature clause chains. As detailed in Rumsey et al. (2020) and Sarvasy (2020) for four children learning Ku Waru and three children learning Nungon, children's first clause chains are attested between 1;9 and 2;9. As with other clause chaining languages for which early productions have been studied to date (Sarvasy \& Choi 2020b), the first Ku Waru and Nungon clause chains are limited to two clauses in length: one medial clause plus one final clause. For both languages, children first produce longer clause chains several months after their first two-clause chain productions, between 2;8 and 3;3. Depending on the child, the first longer clause chains may be limited to three clauses in length, or may range 
from three to five clauses in length. No chains of six or more clauses were attested. Example (1b) shows a Nungon two-clause chain produced by the child TO at age 3;3, in the course of a teasing interchange with her father, about how she will beat her uncle before he can beat her for misbehaving.

(1) a. Father: papa-ya wan $\quad$ wgo=dek honggir-engka- $k=m a-i$.

uncle-2sG.POSS quick PROX=LOC grab-NF-3SG=SUB-TOP

'But your uncle will quickly grab (you) here.'

b. TO:

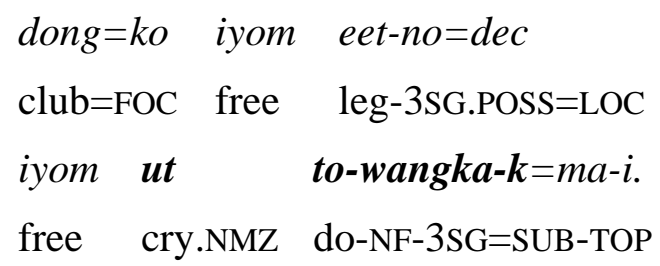

yoo-wa-ya

NSG.O.take-DS.1SG-MV

'But I just taking clubs to his leg, he'll just do crying.'

c. Father: dong=ko irom eet-no=dek $\quad w$-i-ya

club=FOC free leg-3sG.POSS=LOC 3sG.o.beat-DS.2SG-MV

ur-engka-k.

cry-NF-3SG

'You just beating him with a club on his leg, he'll cry.' (TO, 3;3)

TO's father's utterance in (1c) is a rephrasing of her clause chain in (1b). The clause chain itself is well-formed; only two of the verbs are not adult-like, either lexico-semantically ('take them' for 'beat him') or formally ('do crying' for 'cry'; see section 5).

No major patterns of omission (where children omit morphemes or words that adults would include) or commission (where children alter adult forms without omitting them) were identified in clause chain productions: medial verb morphology and ordering of clauses appeared adult-like. Children's first two-clause sentences were clause chains, for both languages: that is, clause chain production preceded that of coordinated or subordinated final clauses. In fact, for Nungon, the patterns for two children are revealing: clause chains and coordinated and subordinated final clause structures are produced at similarly low rates up until about age 3;0, when clause chain tokens begin to take off, while coordinated and subordinated final clause structures increase only slightly, as shown in Figure 3. 


\section{TO's complex sentence tokens (25-39 mos.)}

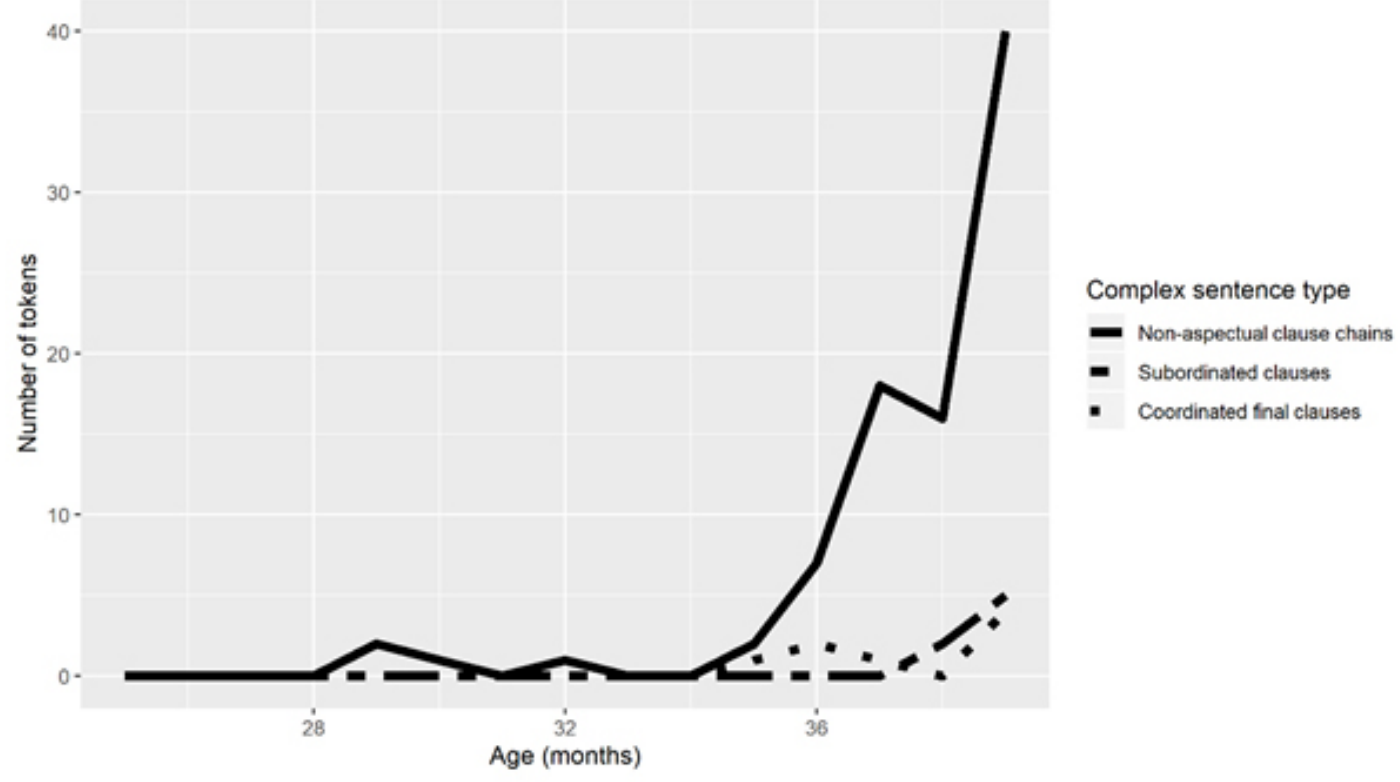

NN's complex sentence tokens (34-39 mos.)

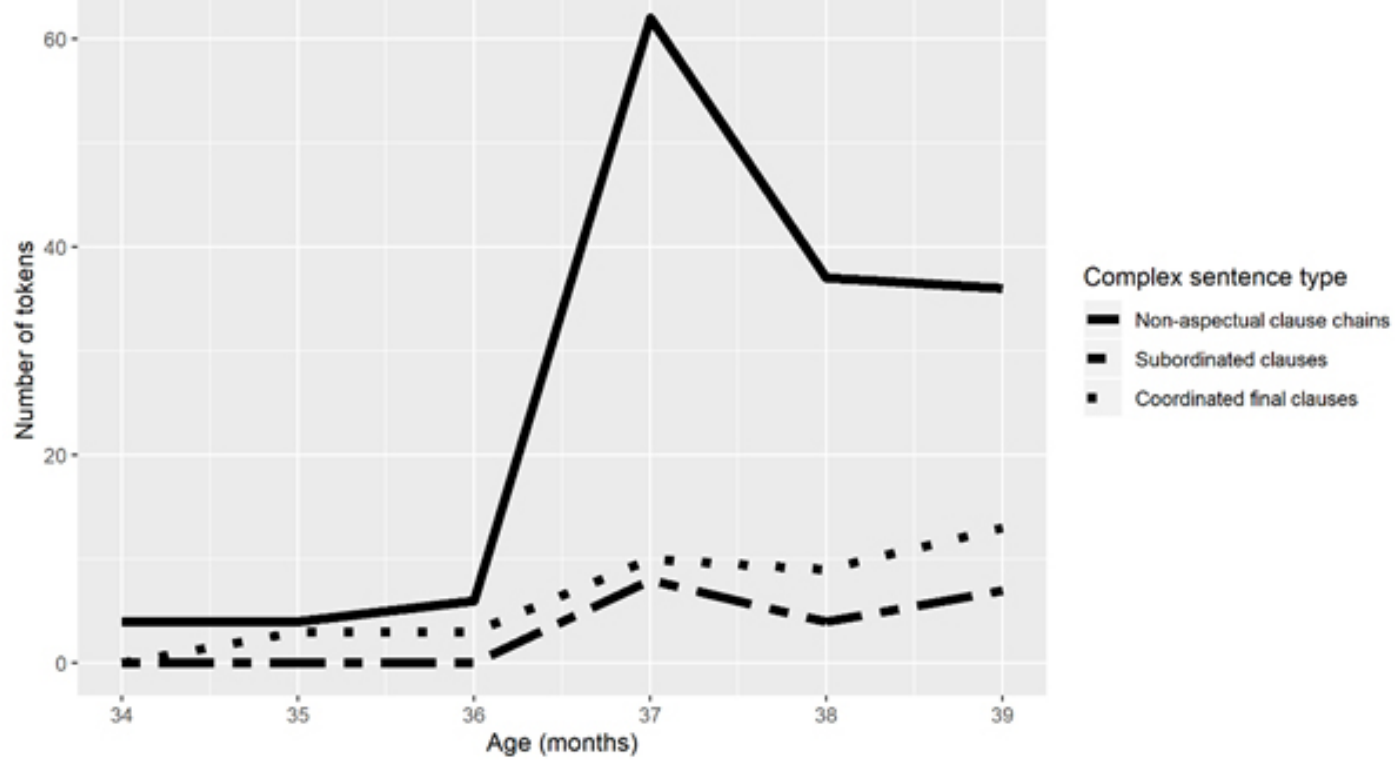

Figure 3. Complex sentence types in the speech of two Nungon-speaking children (Sarvasy 2020)

Children acquiring Ku Waru and Nungon differ dramatically in their production of medial verbs with switch-reference marking indicating 'different-subject'. By age 2;11, both Nungon-speaking children studied at that age were producing two-clause chains with different subject arguments in the first and second clauses, and appropriate switch-reference marking. In fact, 'different-subject' switch-reference marking is also attested much earlier, from 2;1, in incomplete clause chains comprising just the medial clause, or with the medial clause postposed to the final one (see Sarvasy 2015). In contrast, the first productions of medial verbs with different-subject switch-reference marking attested by the Ku Waru- 
speaking children were at 4;7 - two years after the first two-clause chain productions - and only found in the speech of the one child studied at that age. That is, the other three Ku Waru children produced strictly same-subject clause chains throughout the study period, and the oldest child only began producing different-subject clause chains after four-and-a-half.

As Sarvasy (2020) proposed, switch-reference marking on medial verbs in clause chains would seem to pose a cognitive challenge, in that productive use of switch-reference marking requires that a speaker know the identity of the upcoming subject in advance, hence have planned at least part of the upcoming clause before finishing the present clause. Sarvasy suggested that a child learner might have three potential options to deal with this challenge in the initial stages of complex sentence production: a) produce other types of complex sentences, which do not require switch-reference marking, before ever producing clause chains; b) produce only same-subject clause chains for a time, to be able to operate with a 'same-subject' assumption; c) produce morphologically simplified medial verbs, to avoid switch-reference morphology. For both Ku Waru and Nungon, neither (a) nor (c) is attested. Children learning Ku Waru do indeed go through a protracted stage, of over one year, in which their clause chains are limited to same-subject chains. One child learning Nungon (the only one for whom data from the relevant age range were accessible) may have produced only same-subject clause chains for the first five months of clause chain production, but the evidence is inconclusive.

In any case, examination of Ku Waru and Nungon child-directed speech points to another major reason for the dearth of different-subject medial verbs in Ku Waru child speech: Ku Waru adults themselves produced different-subject clause chains sparingly, such that in 5 of 11 sessions sampled, the adults produced no different-subject medial verbs at all, and in the other six sessions, these were always less than $5 \%$ of all medial verbs. In contrast, $10-40 \%$ of all Nungon child-directed medial verbs were different-subject in all 21 sessions analyzed. These differences in child-directed speech point to distributions in the ambient language as being primary in determining how early children begin producing different-subject clause chains. Sarvasy and Choi (2020b) give further comparisons between the development of clause chaining in Ku Waru and Nungon with that in other languages.

\section{Complex predicates}

Many Papuan languages, especially those of the Trans New Guinea grouping (Pawley \& Hammarström 2017) are also known for extensive use of complex predicates. These may take the form of serial verb constructions (see Aikhenvald, this volume); another well-known type of complex predicate involves a light verb-type verbal element and a non-verbal (or nondescript) element, sometimes called an “adjunct” (Pawley \& Hammarström 2017: 112113). In fact, one remarkable characteristic of Nungon child-directed speech (see San Roque \& Schieffelin, this volume) is that simplex, single-verb predicates are optionally expanded into multi-verb predicates involving a nominalized version of the erstwhile lexical verb, supported by a light verb 'do' (Sarvasy 2019): in this quirk of the Nungon child-directed speech style, a simple statement like 'Grandfather died' would become 'Grandfather did dying'; 'look!' would become 'do looking!' This is also true of some child speech, as in (1b) above; there, the child TO literally says 'do crying', which her father rephrases as the adultlike 'cry' in (1c).

Complex predicates like serial verb or verb+adjunct constructions could pose a challenge to the learner because of their phonological and semantic complexity, relative to simple predicates. Further, their meanings are often said to be opaque and lexicalized, such that each 
must be memorized separately. Finally, it might be logical to expect that complex predicates in which one or more elements function grammatically might be acquired differently than those in which all elements contribute lexically. Acquisition of complex predicates has been analyzed for Ku Waru, Nungon and Qaqet. For Ku Waru, for instance, Rumsey (2017) and Merlan and Rumsey (2017) found that children produced adjunct+verb constructions before they produced complex predicates with more than one verb.

Nungon features multi-verb predicates that appear to be serial verb constructions. There are six types of Nungon multi-verb predicates: in the 'general' type, any lexical verb combines with any other(s), but the benefactive, causative, completive aspect, habitual aspect, and imminent aspect multi-verb predicate types involve more restrictions on the last lexical verb, and/or special suffixes on the first lexical verb.

Sarvasy (2021a) analyzed multi-verb predicates in the speech of three children learning Nungon, aged 1;1-2;4, 2;1-3;3, and 2;10-3;3. The youngest child never used multi-verb predicates in the study period; the middle child used them from age 2;4 on, at which age she had not yet mastered all the Nungon tense inflections (as shown in Sarvasy 2019), and the oldest child used them in all sessions. Example (2) shows the first 'general' multi-verb predicate in the speech of the middle child, TO, at 2;4; this follows her parents' pointing out the airstrip on the opposite mountainside:

(2) Nok ö̈̈-ng

PRO.1SG ascend-DEP go-MV.SS

'I going up.' (TO, 2;4)

Later, at 3;2, TO engages in blustering verbal sparring with her mother, and uses the benefactive multi-verb predicate type:

(3)
Eer $-a=d e k$
wee-ya $=$ dek
hai-ng ga-mo-wangka-t.
leg-2SG.POSS=LOC wound-2SG.POSS $=$ LOC
cut-DEP 2SG.O-give-NF-1SG

'I will cut you for you on your wound on your leg!' (TO, 3;2)

Causative multi-verb predicates, which involve two different subject arguments, were rare in child speech; it is unclear whether this primarily stems from morphosyntactic complexity, or discourse context. The middle child showed late development of habitual aspect multi-verb predicates, even though these were robustly present in her parents' child-directed speech. Her uptick in multi-verb predicate use also coincided with an expansion in clause chain length.

Overall, the earliest lexical multi-verb predicates were analyzed as descriptions of multifaceted single actions or events, with later multi-verb predicates also able to describe two or more related actions or events. There was no clear developmental distinction between lexical (Aikhenvald's “symmetrical” serial verb type) and grammatical (Aikhenvald's “asymmetrical” serial verb type) Nungon multi-verb predicate types.

While multi-verb predicates are very common across Papuan languages, there are also languages with other types of complex predicates. This includes Qaqet, which has a large number of complex verbs that are more akin to the prefix and particle verbs of West Germanic (such as uncover or cover up), and usually involve the incorporation of an erstwhile preposition into a semantically-general verb (see also Hellwig, this volume). Hellwig (2020) shows that these complex verbs are relatively rare in the speech of younger children (up to age 2;7), where they occur in fewer than $1 \%$ of utterances (see also Hellwig \& Jung, to appear). These earliest complex verbs are largely repetitions of interlocutors' utterances, deemed unanalyzed chunks. They increase slightly in frequency with age, but 
their numbers still remain low. The attested complex verbs are usually target-like, though, and there is only little overgeneralization. All in all, such complex verbs seem to be acquired fairly late in Qaqet. This is in line with observations from the acquisition of prefix verbs in West Germanic languages (Behrens 1998).

\section{Outlook}

The acquisition of Papuan languages is a greatly under-researched topic, with considerable room for expansion. Currently, the available research covers 8 of the 860 or so Papuan languages. It is an emerging field, though, with a number of on-going research projects. The current focus of research differs in certain crucial aspects from mainstream acquisition research with its grounding in psycholinguistics. This includes especially a very strong affinity to anthropology, language socialization and language documentation, with a focus on spontaneous language use in natural settings. The first experimental studies are emerging, but play a minor role for the moment. Compared to mainstream acquisition research, corpora tend to be much smaller and employ a less dense sampling rate; similarly, participant samples for experimental research tend to be much smaller. In terms of analysis, quantitative analyses are starting to appear (in view of the fact that larger data samples are becoming available), but qualitative analyses continue to play a major role.

These differences are partly due to the methodological challenges that arise when implementing lab-based approaches in fieldwork contexts (see Sarvasy \& Tuninetti, this volume), and partly due to a research tradition that values anthropological perspectives and that encourages community involvement in research design and implementation. Current methodological and theoretical debates center on these issues, aiming to realistically assess the feasibility of language acquisition research on under-documented languages and the contributions it can make to our understanding of language development (Hellwig 2019; Kelly \& Nordlinger 2014). The challenges should not be underestimated, but the available studies show that they can be overcome. Furthermore, there are proposals under way for integrating language acquisition research more firmly into language documentation projects, making it possible to provide 'sketch' acquisition studies on the basis of only 5 hours of analyzed data (Defina et al., in prep.; see also Pye 2021 for a complementary approach).

While corpus construction is a time-consuming endeavor, the resulting corpora constitute long-term resources for investigating the acquisition of varied phenomena. Over the past 5 to 10 years, a number of large-scale acquisition corpora of Papuan languages have become available (see table 2), and most of them are now annotated to the extent that corpus research has become possible. As a direct result, the last few years have seen an increase in the number of acquisition studies, and this trend is likely to continue over the next decades. An achievable mid-term goal will be to consolidate existing research on the acquisition of Papuan languages, adopting a comparative, cross-Papuan, perspective. Successful role models exist, in particular, comparative research on the acquisition of Mayan languages in Mexico (e.g., Pye et al. 2017), or of Bantu languages in Africa (e.g., Demuth 2003).

We would also like to see research into the acquisition of areas related to spoken language, including especially multilingual acquisition (Kulick 1992), but also name tunes in the Yupno-Nankina-Domung area (Amha et al. 2021; Slotta 2012) and signed languages (Reed \& Rumsey 2020). 


\section{Abbreviations}

DEP: dependent; DS: different-subject; FOC: focus; LOC: locative; MV: medial verb; NF: near future; NMZ: nominalizer; O: object; POSS: possessive; PRO: pronoun; PROX: proximate; ss: same-subject; SUB: subordinate; TOP: topicalizer

\section{References}

Aikhenvald, Alexandra Y. 2018. Serial verbs. Oxford: Oxford University Press.

Amha, Azeb, James Slotta \& Hannah S. Sarvasy. 2021. Singing the individual: Name tunes in Oyda and Yopno. Frontiers in Psychology 12: 667599. doi:10.3389/fpsyg.2021.667599

Barton, Michelle E. \& Michael Tomasello. 1994. The rest of the family: The role of fathers and siblings in early language development. In Clare Gallaway \& Brian J. Richards (eds.), Input and interaction in language acquisition, 109-134. Cambridge: Cambridge University Press.

Bavin, Edith L. \& Sabine Stoll (eds.). 2013. The acquisition of ergativity. Amsterdam: John Benjamins.

Casillas, Marisa. In prep. Infants' discrimination of crosslinguistically rare, but native phoneme contrasts in Yélî Dnye.

Casillas, Marisa, Penelope Brown \& Stephen C. \& Levinson. 2017. Casillas HomeBank corpus. doi:10.21415/T51X12

Casillas, Marisa, Penelope Brown \& Stephen C. Levinson. 2020. Early language experience in a Tseltal Mayan village. Child Development 91(5): 1819-1835. doi:10.1111/cdev.13349

Casillas, Marisa, Penelope Brown \& Stephen C. Levinson. 2021. Early language experience in a Papuan community. Journal of Child Language, 48(4): 792-814.

doi:10.1017/S0305000920000549

Casillas, Marisa \& Stephen C. Levinson. In prep. Markedness and minimal pair discrimination in children learning Yélî Dnye.

Cristia, Alejandrina, Emmanuel Dupoux, Michael Gurven \& Jonathan Stieglitz. 2019. Childdirected speech is infrequent in a forager-farmer population: A time allocation study. Child Development 90(3): 759-773. doi:10.1111/cdev.12974

Cristia, Alejandrina \& Marisa Casillas. 2020. LENA recordings gathered from children growing up on Rossel Island. https://gin.g-node.org/EL1000/EL1000

Cutler, Anne. 1985. Cross-language psycholinguistics. Linguistics 23(5): 659-668.

Defina, Rebecca, Birgit Hellwig, Shanley Allen, Lucy Davidson, Barbara F. Kelly \& Evan Kidd. In prep. The Sketch Acquisition Manual. Language Documentation and Conservation, Special Publication.

Demuth, Katherine. 2003. The acquisition of Bantu languages. In Derek Nurse \& Gerard Philippson (eds.), The Bantu languages, 209-222. Surrey: Curzon Press.

Foley, William A. 1986. The Papuan languages of New Guinea. Cambridge: Cambridge University Press.

Frye, Henrike. 2019. Child-directed speech in Qaqet. University of Cologne, PhD Thesis. 
Goldman, Laurence Richard. 1987. Ethnographic interpretations of parent-child discourse in Huli. Journal of Child Language 14(3): 447-466. doi:10.1017/S0305000900010230

Hellwig, Birgit. 2019. A grammar of Qaqet. Berlin: De Gruyter Mouton.

Hellwig, Birgit. 2020. Initial observations on complex predicates in Qaqet children's language. First Language. doi:10.1177/0142723720922004

Hellwig, Birgit, Carmen Dawuda, Henrike Frye \& Steffen Reetz. 2014. Qaqet child language corpus: Longitudinal study. Language Archive Cologne.

Hellwig, Birgit \& Dagmar Jung. 2020. Child-directed language - and how it informs the documentation and description of the adult language. Language Documentation and Conservation 14: 188-214.

Hellwig, Birgit \& Dagmar Jung. To appear. Events of caused accompanied motion in Qaqet and Dëne Sųłné child language corpora. In Anna Margetts, Sonja Riesberg \& Birgit Hellwig (eds.), Caused accompanied motion: Bringing and taking events in a cross-linguistic perspective. Amsterdam: John Benjamins.

Jancso, Anna, Steven Moran \& Sabine Stoll. 2020. The ACQDIV Corpus Database and Aggregation Pipeline. Proceedings of the 12th Conference on Language Resources and Evaluation (LREC 2020), Marseille, 11-16 May, 156-165.

Kelly, Barbara \& Rachel Nordlinger. 2014. Fieldwork and first language acquisition. In Lauren Gawne \& Jill Vaughan (eds.), Selected papers from the 44th Conference of the Australian Linguistic Society, 2013, 178-192.

Kidd, Evan \& Seamus Donnelly. 2020. Individual differences in first language acquisition. Annual Review of Linguistics 6: 319-340. doi:10.1146/annurev-linguistics-011619-03032

Kik, Alfred, Martin Adamec, Alexandra Y. Aikhenvald, Jarmila Bajzekova, Nigel Baro, Claire Bowern, Robert K. Colwell, Pavel Drozd, Pavel Duda, Sentiko Ibalim, Leonardo R. Jorge, Jane Mogina, Ben Ruli, Katerina Sam, Hannah S. Sarvasy, Simon Saulei, George D. Weiblen, Jan Zrzavy, \& Vojtech Novotny. 2021. Language and ethnobiological skills decline precipitously in Papua New Guinea, the world's most linguistically diverse nation.

Proceedings of the National Academy of Sciences 118(22): e2100096118. doi:10.1073/pnas.2100096118

Kulick, Don. 1980. Gapun/Tayap recordings. Collection DK1 at catalog.paradisec.org.au. https://dx.doi.org/10.4225/72/56E979D6D23EA

Kulick, Don. 1992. Language shift and cultural reproduction: Socialization, self, and syncretism in a Papua New Guinea village. Cambridge: Cambridge University Press.

Levinson, Stephen C. To appear. A grammar of Yélî Dnye, the Papuan language of Rossel Island. Cambridge: Cambridge University Press.

Merlan, Francesca \& Alan Rumsey. 2017. Flexibles and polyvalence in Ku Waru: A developmental perspective. In Valentina Vapnarsky \& Edy Veneziano (eds.), Lexical Polycategoriality: Cross-linguistic, cross-theoretical and language acquisition approaches, 307-341. Amsterdam: John Benjamins.

Lieven, Elena V. 1994. Crosslinguistic and crosscultural aspects of language addressed to children. In Clare Gallaway \& Brian J. Richards (eds.), Input and interaction in language acquisition, 56-73. Cambridge: Cambridge University Press. 
Ochs, Elinor. 1988. Culture and language development: Language acquisition and language socialization in a Samoan village. Cambridge: Cambridge University Press.

Pawley, Andrew \& Harald Hammarström. 2017. The Trans New Guinea family. In Bill Palmer (ed.), The languages and linguistics of the New Guinea area: A comprehensive guide, 21-196. Berlin and Bosten: De Gruyter Mouton.

Peute, Abram A. K. \& Marisa Casillas. In revision. Early consonant production in Tseltal and Yélî Dnye.

Pye, Clifton, Barbara Pfeiler \& Pedro Mateo Pedro. 2017. The acquisition of Mayan languages. In Judith Aissen, Nora England \& Roberto Zavala Maldonado (eds.), The Mayan languages, 19-42. London: Routledge.

Pye, Clifton. 2021. Documenting the acquisition of indigenous languages. Journal of Child Language 48: 454-479.

Reed, Lauren W. \& Alan Rumsey. 2020. Sign languages in Papua New Guinea and the Solomon Islands. In Adam Kendon, Sign language in Papua New Guinea: A primary sign language from the Upper Lagaip Valley, Enga Province, 141-183. Amsterdam: John Benjamins. doi:10.1075/z.226.se1

Rispoli, Matthew. 1991. The mosaic acquisition of grammatical relations. Journal of Child Language 18(3): 517-551. doi:10.1017/S0305000900011235

Rumsey, Alan. 1983. Western Highlands of PNG recordings. Collection AR1 at catalog.paradisec.org. https://dx.doi.org/10.4225/72/56E823B109D31

Rumsey, Alan. 2003. Language, desire, and the ontogenesis of intersubjectivity. Language and Communication 23: 169-187.

Rumsey, Alan. 2013. Intersubjectivity, deception and the 'opacity of other minds':

Perspectives from Highland New Guinea and beyond. Language and Communication 33(3): 326-343.

Rumsey, Alan. 2017. Dependency and relative determination in language acquisition: The case of Ku Waru. In Nicholas J. Enfield (ed.), Dependencies in language: On the casual ontology of linguistic systems, 97-117. Berlin: Language Science Press.

Rumsey, Alan. 2019. Intersubjectivity and engagement in Ku Waru. Open Linguistics 5(1): 49-68.

Rumsey, Alan. 2020. Egophoricity, engagement, and the centring of subjectivity. In Henrik Bergqvist \& Seppo Kittila (eds.), Evidentiality, egophoricity, and engagement, 61-93. Berlin: Language Science Press.

Rumsey, Alan, Lauren Reed \& Francesca Merlan. 2020. Ku Waru clause chaining and the acquisition of complex Syntax. Frontiers in Communication 5:19.

doi:10.3389/fcomm.2020.00019

Rumsey, Alan, Lila San Roque \& Bambi Schieffelin. 2013. The acquisition of ergative marking in Kaluli, Ku Waru and Duna (Trans New Guinea). In Edith L. Bavin \& Sabine Stoll (eds.), The acquisition of ergativity, 133-182. Amsterdam: John Benjamin.

Rumsey, Alan et al. In prep. Child-directed speech styles in Ku Waru and Nungon compared.

San Roque, Lila. 2003. Duna (Yuna) recordings. Collection LSR3 at catalog.paradisec.org.au. https://dx.doi.org/10.4225/72/56E97A50C5F4F 
San Roque, Lila \& Bambi B. Schieffelin. 2018. Learning how to know: Egophoricity and the grammar of Kaluli (Bosavi, Trans New Guinea), with special reference to child language. In Simeon Floyd, Elisabeth Norcliffe \& Lila San Roque (eds.), Egophoricity, 437-471. Amsterdam: John Benjamins.

San Roque, Lila \& Bambi B. Schieffelin. 2019. Perception verbs in context: Perspectives from Kaluli (Bosavi) child-caregiver interaction. In Laura J. Speed, Carolyn O’Meara, Lila San Roque \& Asifa Majid. Perception Metaphors, 347-368. Amsterdam: John Benjamins.

Sarvasy, Hannah S. 2015. Breaking the clause chains: Non-canonical medial clauses in Nungon. Studies in Language 39(3). 664-696.

Sarvasy, Hannah S. 2017. Sarvasy Nungon Corpus.

https://childes.talkbank.org/access/Other/Nungon/Sarvasy.html

Sarvasy, Hannah S. 2019. The root nominal stage: A case study of early Nungon verbs. Journal of Child Language 46(6): 1073-1101.

Sarvasy, Hannah S. 2020. Acquisition of clause chains in Nungon. Frontiers in Psychology 11:1456. doi:10.3389/fpsyg.2020.01456

Sarvasy, Hannah S. 2021a. Acquisition of multi-verb predicates in Nungon. First Language. July 2020. doi:10.1177/0142723720938568

Sarvasy, Hannah S. 2021b. On the acquisition of complex predicates: Introduction to the Special Issue. First Language.

Sarvasy, Hannah S. Under review. Prompting of narratives in Nungon child-caregiver conversation.

Sarvasy, Hannah S. \& Soonja Choi (eds.). 2020a. Acquisition of clause chaining. Lausanne: Frontiers Media SA. doi:10.3389/978-2-88966-291-3

Sarvasy, Hannah and Soonja Choi. 2020b. Beyond the two-clause sentence: Acquisition of clause chaining in six languages. Frontiers in Psychology 11:1586.

doi:10.3389/fpsyg.2020.01586

Sarvasy, Hannah, Jaydene Elvin, Weicong Li \& Paola Escudero. 2019. An acoustic analysis of Nungon vowels in adult- versus child-directed speech. In Sasha Calhoun, Paola Escudero, Marija Tabain \& Paul Warren (eds.), Proceedings of the 19th International Congress of Phonetic Sciences, Melbourne, Australia 2019, 3155-3159. Canberra: Australasian Speech Science and Technology Association Inc.

Schieffelin, Bambi B. 1985. The acquisition of Kaluli. In Dan I. Slobin (ed.), The crosslinguistic study of language acquisition, Vol. 1, 525-593. Hillsdale: Erlbaum.

Schieffelin, Bambi B. 1990. The give and take of everyday life: Language socialization of Kaluli children. New York: Cambrige University Press.

Shneidman, Laura \& Amanda L. Woodward. 2016. Are child-directed interactions the cradle of social learning? Psychological Bulletin 142(1): 1-17. doi:10.1037/bul0000023

Slobin, Dan I. (ed.). 1985-1997. The crosslinguistic study of language acquisition, Vol. 1-5. Hillsdale: Erlbaum.

Slotta, James. 2012. On the receiving end: Cultural frames for communicative acts in postcolonial Papua New Guinea. University of Chicago, PhD Thesis. 
Sundara, Megha, Céline Ngon, Katrin Skoruppa, Naomi H. Feldman, Glenda M. Onario, James L. Morgan \& Sharon Peperkamp. 2018. Young infants' discrimination of subtle phonetic contrasts. Cognition 178: 57-66.

Tabain, Marija \& Birgit Hellwig. To appear. Illustration of the IPA: Qaqet. Journal of the International Phonetic Association.

Tabain, Marija, Marc Garellek \& Birgit Hellwig. In prep. Voicing in Qaqet.

Watson-Gegeo, Karen A. \& David W. Gegeo. 1986. Calling-out and repeating routines in Kawara'ae children's language socialization. In Bambi B. Schieffelin \& Elinor Ochs, Language socialization across cultures, 17-50. Cambridge: Cambridge University Press. 